\title{
Targetable ERBB2 mutation status is an independent marker of adverse prognosis in estrogen receptor positive, ERBB2 non- amplified primary lobular breast carcinoma: a retrospective in silico analysis of public datasets
}

Sasagu Kurozumi ${ }^{1,2,3}$, Mansour Alsaleem ${ }^{1,4}$, Cíntia J. Monteiro ${ }^{1,5}$, Kartikeya Bhardwaj ${ }^{1,5}$, Stacey E. P. Joosten ${ }^{6}$, Takaaki Fujii ${ }^{2}$, Ken Shirabe ${ }^{2}$, Andrew R. Green ${ }^{1}$, Ian O. Ellis ${ }^{1}$, Emad A. Rakha ${ }^{1}$, Nigel P. Mongan ${ }^{4}$, David M. Heery ${ }^{5}$, Wilbert Zwart', Steffi Oesterreich ${ }^{7}$ and Simon J. Johnston ${ }^{1,5,8^{*}}$

\footnotetext{
Abstract

Background: Invasive lobular carcinoma (ILC) accounts for 10-15\% of primary breast cancers and is typically estrogen receptor alpha positive (ER+) and ERBB2 non-amplified. Somatic mutations in ERBB2/3 are emerging as a tractable mechanism underlying enhanced human epidermal growth factor 2 (HER2) activity. We tested the hypothesis that therapeutically targetable ERBB2/3 mutations in primary ILC of the breast associate with poor survival outcome in large public datasets.

Methods: We performed in silico comparison of ERBB2 non-amplified cases of ER+ stage I-III primary ILC $(N=279)$ and invasive ductal carcinoma (IDC, $N=1301$ ) using METABRIC, TCGA, and MSK-IMPACT information. Activating mutations amenable to HER2-directed therapy with neratinib were identified using existing functional data from in vitro cell line and xenograft experiments. Multivariate analysis of 10-year overall survival (OS) with tumor size, grade, and lymph node status was performed using a Cox regression model. Differential gene expression analyses by ERBB2 mutation and amplification status was performed using weighted average differences and an in silico model of response to neratinib derived from breast cancer cell lines.

(Continued on next page)
}

\footnotetext{
* Correspondence: simon.johnston@astrazeneca.com

${ }^{1}$ Nottingham Breast Cancer Research Centre, Nottingham Biodiscovery Institute, School of Medicine, University of Nottingham, Nottingham, UK ${ }^{5}$ Gene Regulation and RNA Biology Laboratory, School of Pharmacy, University of Nottingham, Nottingham, UK

${ }^{8}$ Translational Medicine, Oncology Research and Development, AstraZeneca, Darwin Building, Milton, Cambridge, UK

Full list of author information is available at the end of the article
}

C C The Author(s). 2020 Open Access This article is licensed under a Creative Commons Attribution 4.0 International License, which permits use, sharing, adaptation, distribution and reproduction in any medium or format, as long as you give appropriate credit to the original author(s) and the source, provide a link to the Creative Commons licence, and indicate if changes were made. The images or other third party material in this article are included in the article's Creative Commons licence, unless indicated otherwise in a credit line to the material. If material is not included in the article's Creative Commons licence and your intended use is not permitted by statutory regulation or exceeds the permitted use, you will need to obtain permission directly from the copyright holder. To view a copy of this licence, visit http://creativecommons.org/licenses/by/4.0/ The Creative Commons Public Domain Dedication waiver (http://creativecommons.org/publicdomain/zero/1.0/) applies to the data made available in this article, unless otherwise stated in a credit line to the data. 
(Continued from previous page)

Results: ILC tumors comprised $17.7 \%$ of all cases in the dataset but accounted for $47.1 \%$ of ERBB2-mutated cases. Mutations in ERBB2 were enriched in ILC vs. IDC cases $(5.7 \%, N=16$ vs. $1.4 \%, N=18, p<0.0001)$ and clustered in the tyrosine kinase domain of HER2. ERBB3 mutations were not enriched in ILC (1.1\%, $N=3$ vs. $1.8 \%, N=23 ; p=0.604)$. Median OS for patients with ERBB2-mutant ILC tumors was 66 months vs. 211 months for ERBB2 wild-type ( $p=$ 0.0001 ), and 159 vs. 166 months $(p=0.733)$ for IDC tumors. Targetable ERBB2 mutational status was an independent prognostic marker of 10-year OS-but only in ILC (hazard ratio, HR=3.7, 95\% Cl 1.2-11.0; $p=0.021$ ). Findings were validated using a novel ERBB2 mutation gene enrichment score (HR for 10-year OS in ILC $=2.3,95 \% \mathrm{Cl} 1.04-5.05$; $p=0.040)$.

Conclusions: Targetable ERBB2 mutations are enriched in primary ILC and their detection represents an actionable strategy with the potential to improve patient outcomes. Biomarker-led clinical trials of adjuvant HER-targeted therapy are warranted for patients with ERBB2-mutated primary ILC.

Keywords: Lobular, Breast cancer, ERBB2, HER2, Mutation, Prognosis, Therapeutic biomarker, Adjuvant

\section{Background}

Invasive lobular carcinoma of the breast (ILC) accounts for $10-15 \%$ of all breast cancer with an estimated 250, 000 cases per year worldwide [1-4]. Nearly all cases of ILC derive from luminal cells that express estrogen receptor alpha but lack E-Cadherin (CDH1) expression or ERBB2 amplification [5].

Clinical evidence suggests that despite favorable prognostic indicators, e.g., ER+ and/or progesterone receptor positive, low Ki67 proliferation index and HER2- status, patients with ILC have similar or worse long-term outcomes compared to those with invasive ductal carcinoma (IDC, otherwise known as invasive carcinoma of no special type) [5, 6]. Adjuvant treatment of ER+ ILC with letrozole, an aromatase inhibitor, may be superior to tamoxifen, and ILC cells demonstrate resistance to tamoxifen in vitro $[7,8]$. However, patients with ILC are treated according to identical protocols as those with IDC $[9,10]$.

HER2-targeted therapy is indicated for patients whose tumors are HER2+ by immunohistochemistry (IHC) or, if IHC is equivocal, where $E R B B 2$ is amplified as detected by in situ hybridization (ISH) $[11,12]$. Recent evidence indicates that in ERBB2 non-amplified breast cancer, somatic mutation of ERBB2 (ERBB2mut) and/or $E R B B 3$ (ERBB3mut) may provide an alternative mechanism for upregulation of HER2 activity that is therapeutically tractable using second generation HER2 tyrosine kinase inhibitors such as neratinib $[13,14]$.

The epidermal growth factor (EGF) family of receptor tyrosine kinases (HER1-4) are activated by liganddependent homo-/heterodimerisation and regulate cellular proliferation and tumor progression $[15,16]$. In ERBB2-amplified cells, the oncogenic effect of HER2 is mediated by heterodimerisation with HER3 in a ligandindependent manner [17]. Thus HER3 is necessary for HER2 oncogenic activity, and both HER2 and HER3 are therapeutic targets in ERBB2-amplified breast cancer
[18]. Mutations in ERBB2 cluster in the tyrosine kinase and extra-cellular domains of HER2 and exert their oncogenic effects by activating tyrosine kinase activity or increasing HER2 dimerization, respectively [13]. In vitro studies of HER2 activity in cell line and xenograft models identified 13 mutations (listed in the "Patients and methods" section) that enhanced proliferation and/ or demonstrated growth inhibition with the irreversible HER2/EGFR tyrosine kinase inhibitor, neratinib [13]. Mutations in HER3, a critical binding partner for HER2, have been shown to promote ligand (EGF)-independent transformation of breast epithelial cells only in the presence of kinase-active HER2 [19]. This indicates that known oncogenic mutations in ERBB3, e.g., G284R and E928G, may also be therapeutically targetable via HER2 inhibition [19].

$E R B B 2$ mutations have previously been linked with worse prognosis in CDH1-altered ILC: a study of ILC cases in the TCGA dataset $(N=169)$ found that $E R B B 2$ mutations $(N=6)$ occurred exclusively in $C D H 1$-altered tumors $(N=100)$ [20]. However, prognostic data on the $6 E R B B 2$ mut cases were limited to 2 patient events for both disease-free survival and OS analyses. The overall rate of $E R B B 2$ mut in this study of primary ILC was $3.6 \%$ $(N=6$ out of 169$)$. A study of relapsed $C D H 1$-mutated ILC found $E R B B 2$ mut in $18 \%$ of cases $(N=4$ of 22$)$, suggesting further acquisition of $E R B B 2$ mutations in $C D H 1$-altered ILC due to the selective pressure of treatment [21].

To demonstrate the potential clinical benefit of targeting low frequency somatic mutations, prognostic analyses using large clinical datasets are required. In the MA12 trial comprising 328 premenopausal patients with $\mathrm{ER}+$ primary breast cancer of all histological subtypes, non-silent ERBB2 mutations occurred in $5.2 \%$ of patients $(N=17)$ and were adversely prognostic of OS ( $p=$ 0.0114) [22]. A study of 5605 cases of relapsed breast cancer found ERBB2mut in $2.4 \%$ of cases $(N=138)$, of 
which $20 \%(N=27$ of 138$)$ were in ILC tumors [23]. However, neither of these larger studies stratified clinical outcome by histological subtype.

An ILC-specific study of 630 cases of primary ILC found $E R B B 2$ and $E R B B 3$ mutations in 5.1 and $3.6 \%$ of tumors, respectively [24]. Comparison of cases of ER+, HER2 - ILC from the same study $(N=371)$ with cases of ER+, HER2- IDC from TCGA $(N=338)$ indicated significant enrichment of both ERBB2 and ERBB3 mutations in ILC ( 4.3 and $3.5 \%$ in ILC vs. 1.5 and $0.6 \%$ in IDC) [24]. The study reported limited statistical evidence of a time-dependent effect of ERBB2 mutational status associated with short-term breast cancer-specific survival. However, confirmation in datasets including patients with long-term follow-up and decoupling of activating from silent mutations is needed.

We hypothesized that low-frequency somatic mutations in ERBB2 and ERBB3 are enriched in ER+, ERBB2 non-amplified primary ILC cases and may have a demonstrable prognostic effect. We tested these hypotheses by mining a combined dataset of the three largest primary breast cancer series with data on tumor ERBB2 and $E R B B 3$ mutational status, gene expression, clinicopathological features, and patient survival outcomes. Our overall goal was to determine the association between targetable $E R B B 2 / 3$ mutations and survival in primary ER+ ILC, and thereby provide evidence for a clinically actionable strategy to improve patient outcomes.

\section{Patients and methods}

\section{Patients and outcome measures}

Genomic and clinical outcome data associated with tumor samples from patients with primary breast cancer in TCGA $2015(N=817)$, METABRIC 2012 and 2016 $(N=2509)$, and MSK-IMPACT $2018(N=918)$ were accessed online via CBioportal [25-27]. From these datasets, ER+ and HER2- cases of stage I-III ILC and IDC with both clinical outcome and mutational data called from next-generation sequencing (NGS) analyses were selected $(N=1580)$. Cases of mixed or non-ILC/IDC histology, ER-negative/undetermined, HER2+/undetermined, carcinoma in situ, and stage 4/undetermined were excluded. For TCGA and MSK datasets, HER2 status was determined by IHC (positive/negative) or, where IHC was indeterminate, by ISH assessment of ERBB2 amplification, in line with standard clinical practice [11]. For METABRIC cases, HER2 status was determined using the Affymetrix SNP6 copy number inference pipeline.

The primary outcome measure, available in all datasets, was OS. Variables included ERBB2 and ERBB3 mutational status. $E R B B 2$ mut status was subcategorized as oncogenic or uncharacterized by cross-reference with existing data that identified mutations targetable by HER2-inhibition: G309A/E, S310F, L755S, del755-759, S760A, D769H, D769Y, V777L, P780ins, V842I, and R896C [13]. Cases were denominated oncERBB2mut if tumors harbored at least one oncogenic ERBB2 mutation.

Clinical and NGS mutation data were integrated with clinicopathological features including histological subtype, lymph node (LN) status, and tumor size and grade. Normalized gene expression data were publicly available for METABRIC (Illumina HT12 microarray) and TCGA (RNA-seq) datasets.

\section{Statistical analysis}

For analysis of binary somatic mutation status, combined cohort analysis was performed. Enrichment for cases with mutations in candidate genes (ERBB2/3mut) by histological subtype (ILC vs. IDC) was determined by $\chi^{2}$ test for association between categorical variables. For ILC and IDC separately, Kaplan-Meier (KM) survival curves stratified by mutation status were compared using logrank and generalized Wilcoxon tests. Multivariate analysis of OS was performed using a Cox regression model. Covariates included classic prognostic markers tumor grade, size $(<20 \mathrm{~mm}$ or $\geq 20 \mathrm{~mm})$, and $\mathrm{LN}$ status (positive or negative). Adjustment was made for age at diagnosis ( $<50$ or $\geq 50$ years). Tumor grade was classified as low (grade 1-2) or high (grade 3).

To derive a novel gene expression signature of HER2 activity that accounted for the effect of potentially targetable ERBB2mut in ERBB2 non-amplified tumors, we applied a weighted average difference (WAD) method to gene expression data in cases from the METABRIC 2012 $(N=1980)$ and TCGA $2015(N=817)$ cohorts [25, 28, 29]. Gene expression in ERBB2mut cases $(N=38$, selected by $E R B B 2$ non-amplified status and patient age > 50) was compared with the same order of magnitude of $E R B B 2$ wild-type cases $(N=79$, selected by ERBB2 nonamplified status, grade $>1$, stage $>\mathrm{I}$, patient age $>50)$. This was repeated for oncERBB2mut cases $(N=23)$ using the same comparator and selection criteria. To incorporate the effect of HER2 activity via ERBB2 amplification, the overlap of differentially expressed genes DEGs shared by both comparisons (ERBB2mut and oncERBB2mut vs. ERBB2 wild-type) with DEGs from a further comparison of ERBB2 amplified $(N=247)$ vs. non-amplified $(N=1733)$ cases in METABRIC was calculated. Finally, to incorporate the downstream phenotype (HER2 status), the overlap of this list with DEGs from a comparison of clinical HER2+ vs. HER2- cases in TCGA was calculated. In contrast to ERBB2mut cases, matching was not performed for ERBB2 amplified or HER2+ cases because numbers were higher and within an order of magnitude across groups, such that 
similar variation in gene expression could reasonably be assumed.

Multiple gene expression signatures of HER2 activity have been derived using cell line models and patient tumors [30-33]. We compared our novel gene signature with the HER2 activity signature established by Desmedt et al [31] with respect to its ability to detect potentially targetable ERBB2mut cases in our ILC/IDC dataset. This was achieved by multivariate regression modeling of response to neratinib for each gene signature using breast cancer cell line pharmacogenomic data from the BROAD Institute, accessed online via the CellMinerCDB portal $[34,35]$. The Pearson coefficient for each significantly correlated signature gene was used to calculate normalized signature scores for each METABRIC case in the current dataset. To validate the prognostic effect of $E R B B 2$ in ILC, cases were then stratified by gene signature score (upper vs. lower quartiles) and the signatures compared by histological subtype using a Cox regression model of a 10-year OS.

\section{Results}

Clinicopathological landscape of the combined cohort

All cases of primary (stage I-III) ER+ and HER2ILC and IDC from METABRIC, TCGA, and MSK-
IMPACT cohorts were selected (total $N=1580$, see Table 1). Baseline clinicopathological characteristics by individual cohort are summarized in Table 1 .

To detect prognostic effects of low-frequency mutations and add to the existing body of literature on ILCspecific mutational drivers, a combined cohort of three public datasets was collated. We first evaluated the implications of combining cases of ILC and IDC from potentially disparate datasets.

In the combined cohort, long-term follow-up of patients is dominated by the largest dataset (METABRIC, $N=702$ : mean follow-up 133 months) while early events are enriched by the two smaller datasets (TCGA and MSK, $N=878$ : mean follow-up 33 months) (see Supplemental Figure S1(A) in Additional file 1 for KM plot). Compared to the smaller datasets with shorter follow-up (TCGA and MSK), patients in METABRIC were more likely to be over 50 years of age and have T1 tumors $(<$ $20 \mathrm{~mm}$ diameter), but no significant difference in grade or LN status was found (see Table 2). As the principle skew in the combined dataset is towards longer followup in METABRIC cases, OS analysis was limited to 10 years-thus providing a clinically meaningful endpoint for all patients, irrespective of age. Comparison of

Table 1 Baseline clinicopathological characteristics of the combined cohort

\begin{tabular}{|c|c|c|c|c|c|c|c|c|c|}
\hline & & \multicolumn{2}{|c|}{ METABRIC $(N=702)$} & \multicolumn{2}{|c|}{ TCGA $(N=330)$} & \multicolumn{2}{|c|}{ MSK-IMPACT $(N=548)$} & \multicolumn{2}{|c|}{ Total $(N=1580)$} \\
\hline & & $N$ & $\%$ & $N$ & $\%$ & $N$ & $\%$ & $N$ & $\%$ \\
\hline \multirow[t]{2}{*}{ Histology* } & ILC & 76 & 10.8 & 100 & 30.3 & 103 & 18.8 & 279 & 17.7 \\
\hline & IDC & 626 & 89.2 & 230 & 69.7 & 445 & 81.2 & 1301 & 82.3 \\
\hline \multirow[t]{2}{*}{ Age } & $<50$ years & 125 & 17.8 & 87 & 26.4 & 190 & 34.7 & 402 & 25.4 \\
\hline & $\geq 50$ years & 577 & 82.2 & 243 & 73.6 & 358 & 65.3 & 1178 & 74.6 \\
\hline \multirow[t]{3}{*}{ Menopause } & Pre- & 125 & 17.8 & 90 & 27.2 & 234 & 42.7 & 449 & 28.5 \\
\hline & Post- & 577 & 82.2 & 219 & 66.4 & 309 & 56.4 & 1105 & 69.9 \\
\hline & unknown & 0 & 0 & 21 & 6.4 & 5 & 0.9 & 26 & 1.6 \\
\hline \multirow[t]{3}{*}{ Stage $^{*}$} & । & 238 & 33.9 & 67 & 49.3 & 270 & 49.3 & 575 & 36.4 \\
\hline & $\|$ & 418 & 59.5 & 188 & 34.3 & 188 & 34.3 & 794 & 50.3 \\
\hline & III & 46 & 6.6 & 75 & 16.4 & 90 & 16.4 & 211 & 13.4 \\
\hline \multirow[t]{2}{*}{ Tumor size } & $<20 \mathrm{~mm}$ & 215 & 30.6 & 96 & 29.1 & 330 & 60.2 & 641 & 40.6 \\
\hline & $\geq 20 \mathrm{~mm}$ & 487 & 69.4 & 234 & 70.9 & 218 & 39.8 & 939 & 59.4 \\
\hline \multirow[t]{4}{*}{ Tumor grade } & 1 & 61 & 8.7 & 49 & 14.8 & 51 & 9.3 & 161 & 10.2 \\
\hline & 2 & 344 & 49.0 & 190 & 57.6 & 191 & 34.9 & 725 & 45.9 \\
\hline & 3 & 268 & 38.2 & 81 & 24.5 & 288 & 52.6 & 637 & 40.3 \\
\hline & Unknown & 29 & 4.1 & 10 & 3.0 & 18 & 3.3 & 57 & 3.6 \\
\hline \multirow[t]{3}{*}{ Follow-up } & $<5$ years & 137 & 19.5 & 261 & 82.8 & 454 & 82.8 & 852 & 53.9 \\
\hline & $5-10$ years & 195 & 27.8 & 61 & 11.7 & 64 & 11.7 & 320 & 20.3 \\
\hline & $\geq 10$ years & 370 & 52.7 & 8 & 5.5 & 30 & 5.5 & 408 & 25.8 \\
\hline \multirow[t]{2}{*}{ Status } & Alive ${ }^{* *}$ & 310 & 44.2 & 299 & 90.1 & 494 & 90.1 & 1103 & 69.8 \\
\hline & Deceased & 392 & 55.8 & 31 & 9.9 & 54 & 9.9 & 477 & 20.3 \\
\hline
\end{tabular}

${ }^{*}$ Cases of ILC/IDC histology, stage I-III, ER+ and HER2 - status with clinical outcome and mutational data were selected via CBioportal 
Table 2 Comparison of long vs. short follow-up cohorts: METABRIC (largest dataset, long follow-up) vs. TCGA and MSK (combined smaller datasets, short follow-up). Significant difference was found with respect to age and tumor size, but not tumor grade or LN status. Table excludes "unknown" cases for each variable

\begin{tabular}{|c|c|c|c|c|c|c|}
\hline & & \multicolumn{2}{|c|}{ METABRIC $(N=702)$} & \multicolumn{2}{|c|}{ TCGA AND MSK $(N=878)$} & \multirow[t]{2}{*}{$x^{2}$ test } \\
\hline & & $N$ & $\%$ & $N$ & $\%$ & \\
\hline \multirow[t]{2}{*}{$\overline{\text { Age }}$} & $<50$ years & 125 & 17.8 & 277 & 31.5 & \multirow[t]{2}{*}{$p<0.00001$} \\
\hline & $\geq 50$ years & 577 & 82.2 & 601 & 68.5 & \\
\hline \multirow[t]{2}{*}{ Tumor size } & $<20 \mathrm{~mm}$ & 215 & 30.6 & 426 & 48.5 & \multirow[t]{2}{*}{$p<0.00001$} \\
\hline & $\geq 20 \mathrm{~mm}$ & 487 & 69.4 & 452 & 51.5 & \\
\hline \multirow[t]{2}{*}{ Tumor grade } & $1-2$ & 405 & 60.2 & 481 & 56.6 & \multirow[t]{2}{*}{$p=0.158$} \\
\hline & 3 & 268 & 39.8 & 369 & 43.4 & \\
\hline \multirow[t]{2}{*}{ LN status } & Negative & 370 & 53.9 & 489 & 55.8 & \multirow[t]{2}{*}{$p=0.457$} \\
\hline & Positive & 316 & 46.1 & 387 & 44.2 & \\
\hline
\end{tabular}

METABRIC with combined TCGA and MSK-IMPACT cohorts revealed no significant difference in a 10-year OS (logrank $p=0.225$, see Supplemental Figure S1(B) in Additional file 1).

\section{ERBB2 mutations are enriched in primary ILC and cluster in the HER2 tyrosine kinase domain}

We next assessed the prevalence of $E R B B 2 / 3$ mut in our dataset of ILC and IDC $(N=1580)$. Overall prevalence of ERBB2mut was $2.2 \%(N=34)$. ERBB2mut was enriched in ILC, with prevalence of $5.7 \%(N=16)$ vs. $1.4 \%$ in IDC $(N=18)(p<0.0001)$. In contrast, prevalence of ERBB3mut was lower (1.6\% overall, $N=26)$, and there was no enrichment of $E R B B 3 \mathrm{mut}$ in ILC vs. IDC $(1.1 \%, N=3$ vs. $1.8 \%, N=23 ; p=0.604)$. Due to the small number of cases in ILC, further analysis of ERBB3mut was not performed.

In ILC, ERBB2mut clustered in the tyrosine kinase domain of HER2 (15 out of 16 cases; 93.8\%). Of these, the majority have been characterized as oncogenic and potentially targetable using neratinib (oncERBB2mut, 11 out of 15 kinase domain mutations in ILC; 73.3\%). In IDC, all kinase domain mutations were known oncogenic $(N=8)$ and non-characterized mutations were distributed evenly across protein domains (see Fig. 1). The most frequently occurring ERBB3mut coded for E928G, which lies in the tyrosine kinase domain of HER3 ( $N=7$ out of $26 ; 27 \%$ ).

ERBB2 mutation is an adverse prognostic indicator of survival for patients with primary ILC

Using our meta-cohort of 1580 cases, we next assessed $E R B B 2$ mut as a prognostic marker of OS in ILC ( $N=$ 279) and IDC $(N=1301)$. Median duration of patient follow-up was 50 months (range 0-351 months). In patients with ILC, median OS was significantly shorter if tumors were $E R B B 2$ mut positive (inclusive of oncogenic and uncharacterized mutations) vs. ERBB2mut negative (66 vs. 211 months, $p=0.0001$ ). In contrast, there was no significant difference in OS for $E R B B 2$ mut cases of IDC (159 vs. 166 months, $p=0.733$ ) (see Fig. 2).

$E R B B 3$ mut status was not a significant prognostic indicator of $\mathrm{OS}$ in cases of IDC $(\mathrm{HR}=1.46,95 \%$ CI $0.65-$ $3.28 ; p=0.359$ ). There were no events (patient deaths) at 10 years of follow-up in the three cases of ERBB3mut ILC.

Targetable ERBB2 mutation status is an independent adverse prognostic marker of 10-year overall survival in ER+, ERBB2 non-amplified ILC

To test the effect of therapeutically actionable ERBB2 mutations on a clinically relevant endpoint, we stratified 10 -year OS by oncERBB2mut status. In ER+, ERBB2 non-amplified ILC, oncERBB2mut status was prognostic of 10-year OS independently of LN status, tumor grade, and size (HR 3.65, 95\% CI 1.21-11.00; $p=0.021$, see Fig. 3). The independent prognostic value of oncERBBmut status on 10-year OS was retained after further adjusting for age at diagnosis (HR 3.19, 95\% CI 1.069.62; $p=0.039$ ).

Unselected ERBB2mut status (including oncogenic and uncharacterized mutations) was also adversely prognostic of 10-year OS in univariate analysis (HR 3.66, 95\% CI 1.54$8.73 ; p=0.003)$, but in contrast to characterized oncERBB2mut, this prognostic effect was not independent of LN stage, tumor grade, and/or size. The independent prognostic value of oncERBB2mut was further verified at 5 years of follow-up (HR 3.668, 95\% CI 1.096-12.275; $p=0.035$ ).

\section{A novel gene signature of targetable HER2 activity using ERBB2mut status}

Since existing gene signatures of HER activity derive from HER2 status (by IHC and/or ISH), we generated a novel gene signature incorporating DEGs in ERBB2mut and oncERBB2mut cases, as described in the "Patients and methods" section and outlined in Fig. 4. Our aim was to establish a novel signature that reflects HER2 
(A) Proportion of cases by histology: $\square$ ILC $\square$ IDC



(B) ERBB2mut
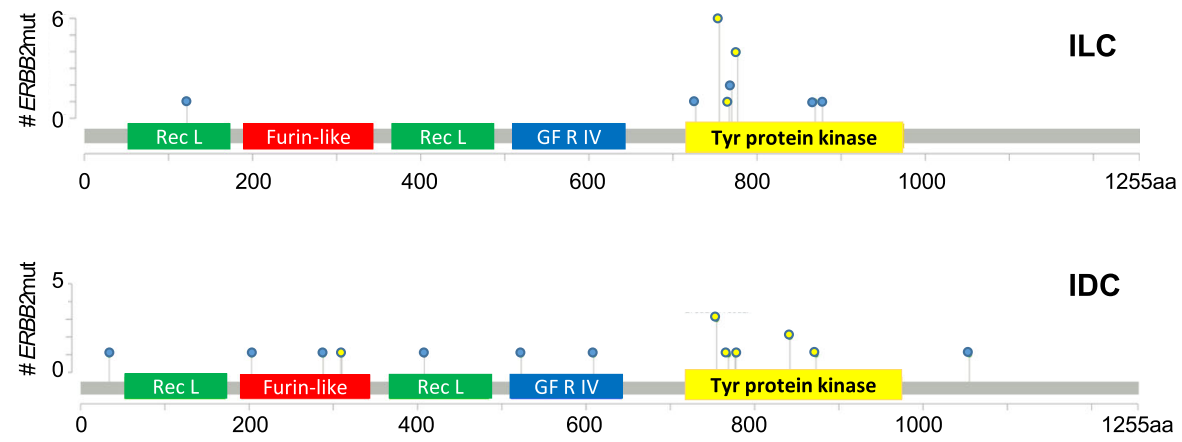

(C) ERBB3mut
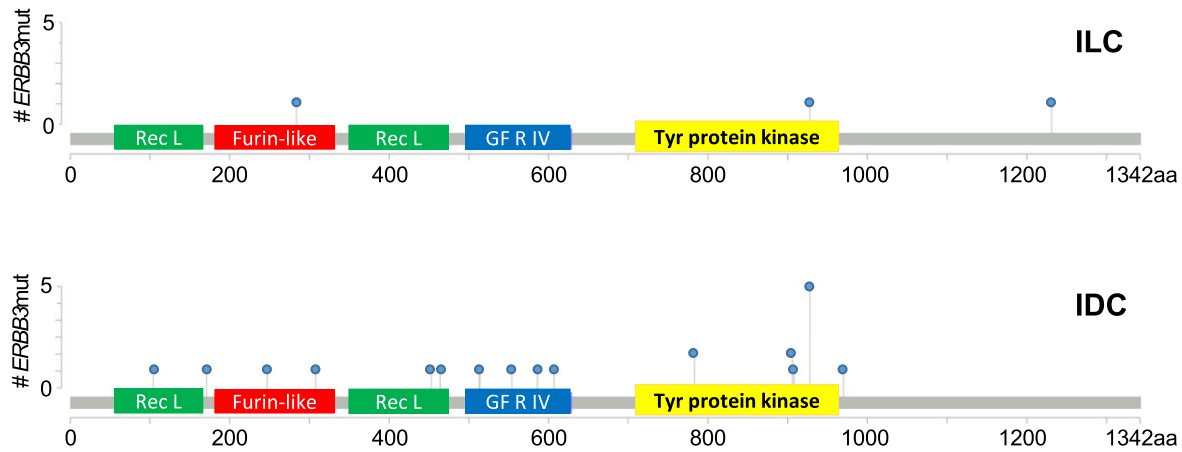

Fig. 1 Rate of ERBB2 and ERBB3 mutations and their spatial distribution on HER2/3 in ILC and IDC. ERBB2mut were found to be a enriched in ILC (yellow bar) vs. IDC (blue bar) and $\mathbf{b}$ clustered in the tyrosine kinase domain of HER2; c ERBB3mut occurred at lower frequency, with the highfrequency outlier in IDC coding for known oncogenic HER3 kinase domain alteration E928G $(N=6)$. Y-axes show the number of cases harboring at least one ERBB2/3 mutation at a specific amino acid (aa) of HER2/3, shown along the $x$-axes. Yellow-filled circles indicate oncERBB2mut. Extracellular domains of HER2/3: Receptor L, Furin-like and Growth Factor Receptor IV; intracellular: tyrosine protein kinase. ${ }^{*} p<0.001$; $\mathrm{n} / \mathrm{s}=$ not significant

pathway activity more completely than existing signatures, whether induced by ERBB2 mutations or amplifications, and to be able to apply it in a wider range of patients. A list of up and downregulated ERBB2 "mutant" DEGs $(N=20)$ was generated by combining the overlap between DEGs for METABRIC ERBB2 amplified vs. non-amplified, $E R B B 2$ mutated vs. wild-type (ERBB2mut and oncERBB2mut separately), and TCGA HER2+ vs. HER2- (Fig. 4a, and see Additional file 2 for Supplemental Tables S1-3 with all gene lists).
As shown in Fig. 4b, ERBB2mut and oncERBB2mut clustered in the upper quartile of the novel gene signature score in cases of ER+, HER2- ILC/IDC from METABRIC. In contrast, ERBB2mut and oncERBB2mut cases did not cluster when scored by the established gene signature of HER2 pathway activation but were evenly distributed across the cohort. Both the novel and established HER2 pathway gene signatures were predictive of response to neratinib in multivariate analysis using a pharmacogenomic breast cancer cell line model 


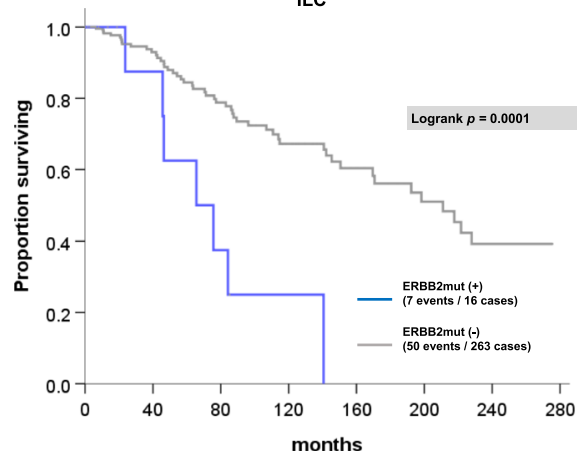

IDC

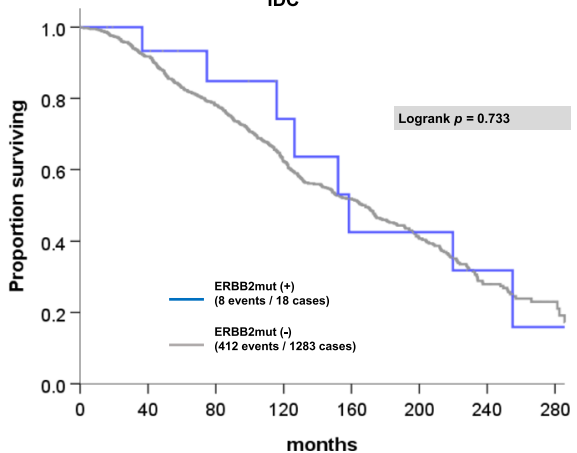

Fig. 2 OS by ERBB2 mutational status in ILC (left) and IDC (right). Gray line indicates ERBB2 wild-type cases; blue line indicates cases with at least one ERBB2mut

(Pearson $R=0.9, p<1 \mathrm{e}^{-14}$ for both; see Supplemental Figure S2 in Additional file 3 for the original plots from the CellMinerCDB online portal). The regression model used incorporates drug response and gene expression data from $N=36$ breast cancer cell lines [35, 36]. Our findings using this model verify that the novel signature derived from DEGs in ERBB2mut vs. wild-type cases incorporates oncogenic HER2 activity due to ERBB2mut. This suggests that a gene signature may have value in predicting targetable ERBB2 alterations including $E R B B 2$ mut in HER2- breast cancer. Finally, Fig. 4c demonstrates that the novel gene signature score (stratified into upper vs. lower quartiles) was adversely associated with 10-year OS (HR $=2.3,95 \%$ CI 1.04-5.05; $p=0.040)$, thus providing mRNA level validation of ERBB2mut as an adverse prognostic marker.

Using the same dataset (CellMinerCDB), it was not possible to associate ERBB2mut status with neratinib response as only one breast cancer cell line (MDA-MB468) was $E R B B 2$ mut positive. Response to neratinib for MDA-MB-468 was in the upper tertile of breast cancer cell lines (data in the public domain via CellMinerCDB online portal).

\section{Discussion}

In this study, we mined clinical and NGS data from the largest clinical cohorts with data in the public domain to test whether there was an ILC-specific association of $E R B B 2$ mutations with OS. In our meta-cohort of ER+, $E R B B 2$ non-amplified cases of ILC and IDC, we found that ERBB2 mutations are enriched ILC, cluster in the functional kinase domain of HER2, and robustly associate with adverse clinical outcomes-independently of known prognostic clinicopathological features including LN status and tumor grade. In contrast, there was no ILC-specific enrichment of ERBB3mut.

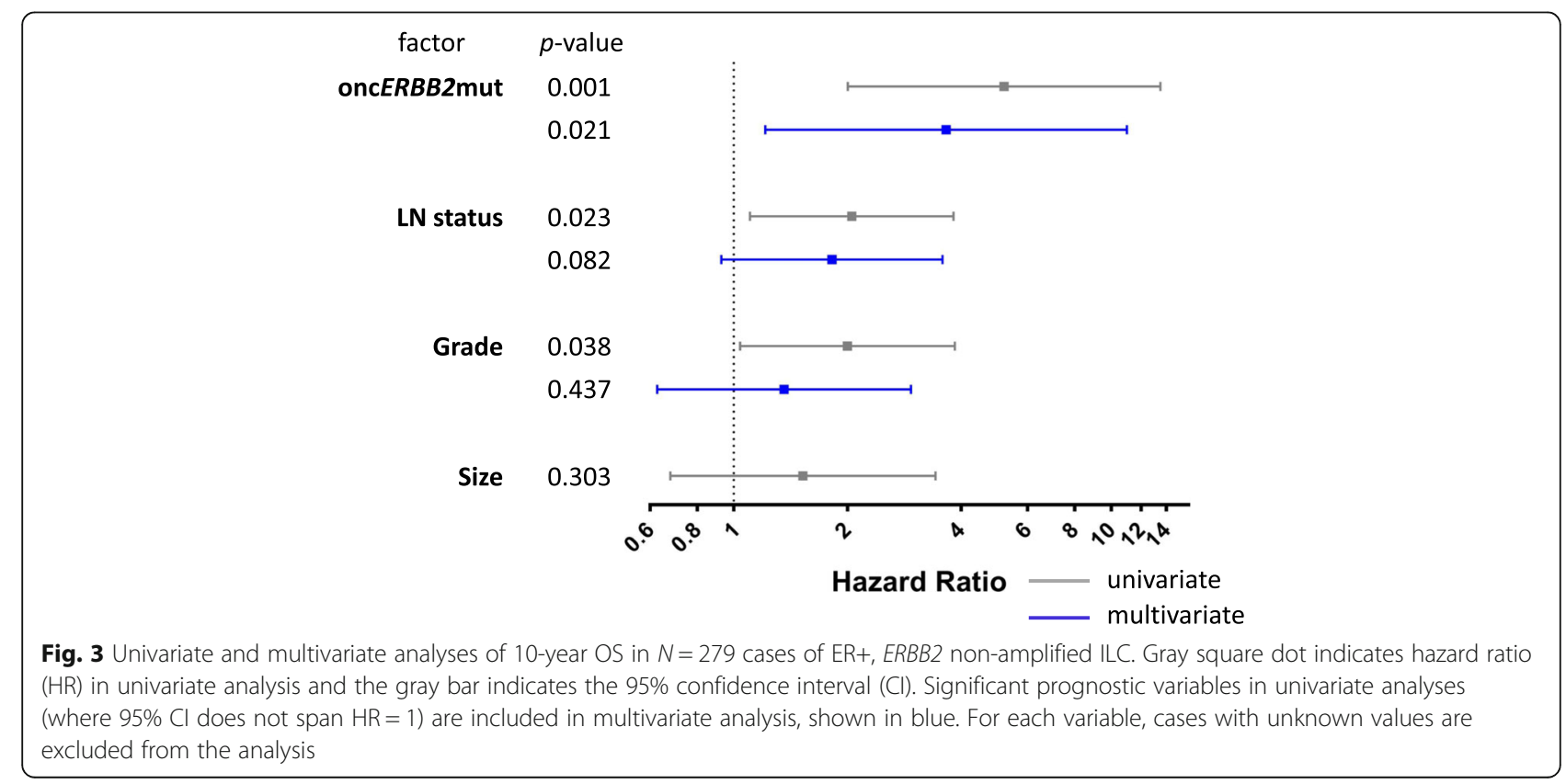




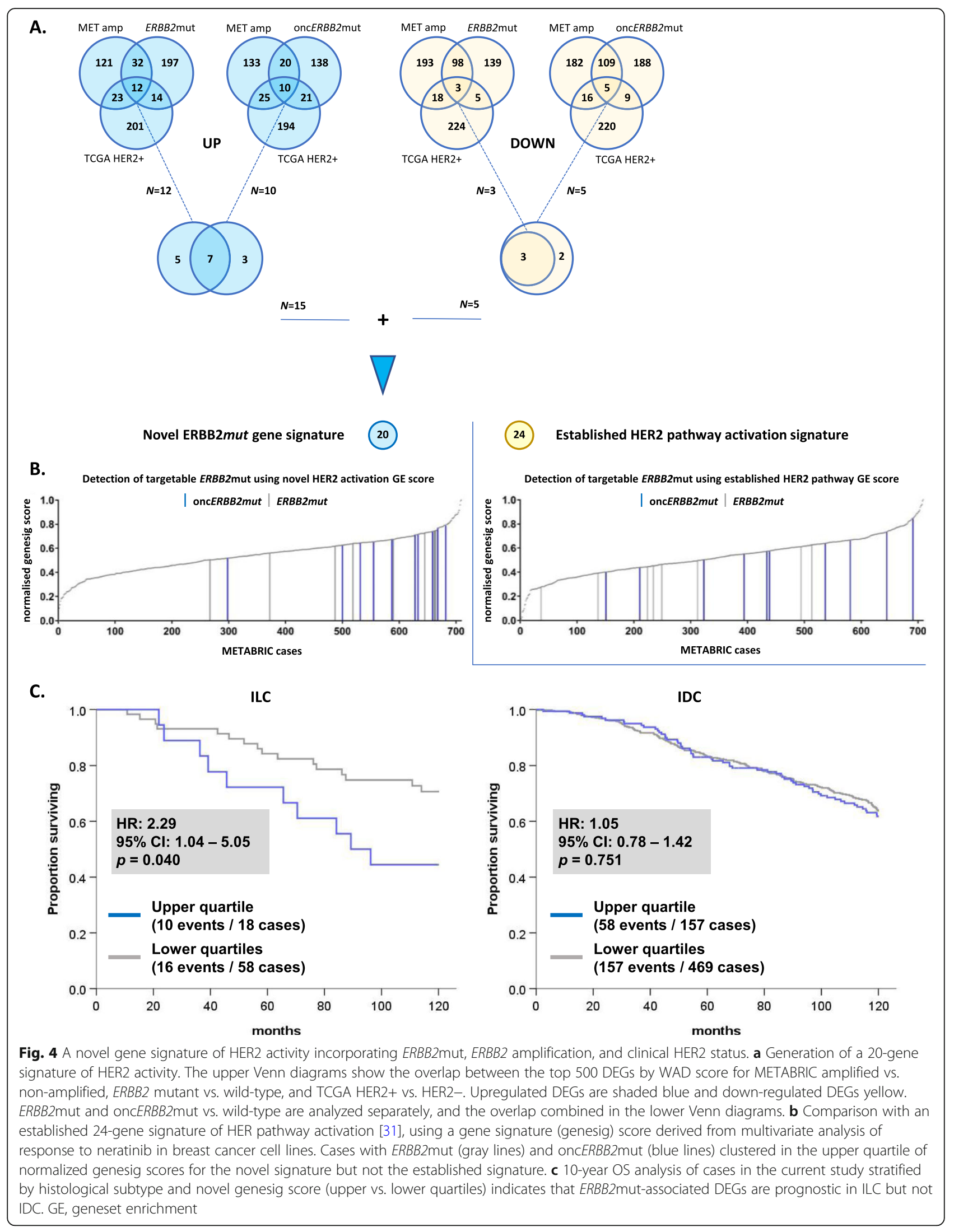


Compared to the largest previous study with data on $E R B B 2$ mutational status in primary ILC $(N=371)$ [24], the current study found higher frequency of $E R B B 2$ mut (5.7 vs. $4.3 \%$ ) but lower frequency of ERBB3mut (1.1 vs. 3.5\%). Hazard proportionality in the study by Desmedt et al 2016 suggested that $E R B B 2$ mutational status had a time-dependent effect associated with short-term risk of breast cancer relapse [24]. The current study adds data on the prognostic effect of specific ERBB2 mutations that were previously shown in cell line, in vitro and xenograft studies to respond to existing clinical compounds such as trastuzumab or neratinib [13]. We demonstrated that the status of these therapeutically targetable ERBB2 mutations is an independent adverse prognostic marker of overall survival in a large cohort of patients with ILC.

Findings from the current study imply that targeted sequencing of ILC tumors for ERBB2 mutations may be an actionable and viable strategy to improve outcomes for patients with primary ILC by providing a biomarker for HER2-targeted therapy in the adjuvant setting. Based on worldwide ILC incidence of 250,000 [1-4], 10\% rate of ER- and/or HER2- status in ILC, and $5 \%$ rate of potentially targetable ERBB2mut in $\mathrm{ER}+, E R B B 2$ non-amplified ILC, we conservatively estimate that 11,250 additional ILC patients per year who have ERBB2-mutated tumors may benefit from HER2-targeting therapy.

Existing clinical studies of HER2-targeting therapy are underway in advanced breast cancer. For example, neratinib (alone or in combination with fulvestrant) was tested in phase II study for patients with advanced, ERBB2 non-amplified, ERBB2mut breast cancer (clinical trial registration number NCT01670877). Of the 16 patients who had evaluable tumor responses, $N=2$ had partial response by RECIST criteria (13\%). Out of 14 patients with tumors harboring oncERBB2mut, $N=5$ (36\%) derived clinical benefit, defined as stable disease or partial/complete response by RECIST criteria [37]. Given that the patients in this trial had received multiple prior lines of treatment, it is conceivable that patients with ERBB2 nonamplified primary tumors harboring oncERBB2mut will also derive benefit from (neo) adjuvant HER2targeted therapy such as neratinib.

In the current cohort of primary ER+, HER2- ILC and IDC, ERBB2 mutations in ILC tumors clustered almost exclusively in the active HER2 tyrosine kinase domain $(N=15$ of 16$)$. Our retrospective clinical outcome data indicates an ILC-specific adverse survival association for patients with $E R B B 2$ non-amplified tumors harboring potentially targetable ERBB2 mutations. This primary finding was validated by existing
mRNA data via a novel gene signature of HER2 activity, which linked ERBB2mut with response to neratinib. Subject to independent external validation, a gene signature of HER2 activity could have clinical application as a biomarker of response to HER2targeted small molecules such as neratinib and may be more cost-effective than targeted NGS in the detection of $E R B B 2$ mut in primary ILC.

Taken together, these new findings suggest that focusing early phase studies of HER2-inhibition (e.g., with neratinib) in patients with $E R B B 2$ non-amplified, $E R B B 2$ mut primary ILC may be an effective strategy to demonstrate feasibility and clinical benefit in the (neo) adjuvant setting.

Limitations of the current study include cohort size, quality of clinical outcome data, and bias in longterm follow-up towards cases from the largest dataset (METABRIC). Statistical power is limited by the small number of patient deaths in cases of ILC and IDC with $E R B B 2$ mut ( $N=7$ and 8, respectively). However, the clear deviation of the KM plot of OS in ILC stratified by $E R B B 2 \mathrm{mut}$ (Fig. 2) is reflected in a low type I error of one in $10,000(p=0.0001)$. In contrast, there was no ILC-specific enrichment of ERBB3mut in our database. However, in IDC there was enrichment of the known oncogenic HER3 kinase domain mutation, E928G. To demonstrate the prognostic effect of mutations affecting HER3, larger datasets will be required.

The effect of incomplete clinical outcome data on the primary endpoint (OS) is difficult to quantify but is accounted for statistically in Cox regression modeling by censoring patients at last follow-up. Patients in METABRIC presented at older age, which is associated with more indolent, less biologically aggressive breast cancer [38]. This implies an underestimation of the adverse impact of ERBB2 mutations on 10-year survival outcomes in the current study. Since the METABRIC dataset did not have complete HER2 IHC or reverse-phase protein array data, it was not possible to associate activating $E R B B 2$ mut directly with downstream HER2 protein expression. Instead, we generated a gene signature incorporating clinical HER2 status to validate our findings.

\section{Conclusions}

Targetable kinase domain ERBB2 mutations are enriched in primary ILC and their detection by targeted sequencing or validated gene signature surrogate may provide an actionable strategy to improve patient outcomes. Biomarker-led clinical trials of adjuvant HER2-targeted therapy to treat breast tumors with activating ERBB2 mutations are warranted in HER2- primary ILC. 


\section{Supplementary information}

Supplementary information accompanies this paper at https://doi.org/10. 1186/s13058-020-01324-4.

Additional file 1: Supplemental Figure S1. (A) 10-year OS KM plot stratified by individual study cohort: blue - METABRIC, solid gray - TCGA, dotted gray - MSK-IMPACT; (B) 10-year OS KM plot stratified by study category: blue - long follow-up (METABRIC), gray - short follow-up (TCGA and MSK-IMPACT combined); Logrank $p=0.225$.

Additional file 2: Supplemental Tables. (S1) list of genes in the novel $(N=20)$ and established (Desmedt et al [31], $N=24)$ gene signatures of HER2 activity; (S2 and S3) top 500 DEGs by WAD score stratified into genes that are up-regulated (S2) and down-regulated (S3) for METABRIC ERBB2 amplified vs. non-amplified (ERBB2amp), METABRIC ERBB2mut vs. wild-type (ERBB2mut) and oncERBB2mut vs. wild-type (oncERBB2mut), and TCGA HER2+ vs. HER2- (TCGA HER2+).

Additional file 3: Supplemental Figure S2. Correlation between the observed response of breast cancer cell lines $(N=36)$ to neratinib and the response predicted by expression of $(A)$ novel $(N=20)$ and $(B)$ established (Desmedt et al [31], $N=24$ ) gene panels. Plots were generated using CellMinerCDB online portal and cell line data from the BROAD Institute [34, 35].

\section{Abbreviations}

Cl: Confidence interval; DEG: Differentially expressed gene; EGF: Epiderma growth factor; ER: Estrogen receptor alpha; ERBB2/3mut: ERBB2/3 mutant; HER2/3: Human epidermal growth factor receptor 2/3; HR: Hazard ratio; IDC: Invasive ductal carcinoma of the breast; IHC: Immunohistochemistry; ILC: Invasive lobular carcinoma of the breast; ISH: In situ hybridization; KM: Kaplan-Meier; LN: Lymph node; NGS: Next-generation sequencing; oncERBB2mut: Oncogenic ERBB2 mutant; OS: Overall survival; RECl ST: Response evaluation criteria in solid tumors; WAD: Weighted average difference

\section{Acknowledgements}

Not applicable

\section{Authors' contributions}

SK, DMH, WZ, SO, and SJJ conceived, designed, and supervised the study. SK, MA, CM, KB, NPM, and SJJ analyzed and interpreted the data. SK, WZ, SO, and SJJ prepared the manuscript. ARG, IOE, and EAR had supervisory and administrative roles. SJ, TF, and KS had consultative roles in study design. All authors were involved in manuscript preparation and reviewing for submission, gave their final approval, and agreed to be accountable for all aspects of the work.

\section{Funding}

The corresponding author (SJJ) is funded by the Wellcome Trust (Royal Academy of Medical Sciences grant number AAM 127669) and the National Institute for Health Research UK. Dr. Oesterreich's work on ILC is supported by the Breast Cancer Research Foundation and a Komen Scholar awards (SAC160073).

\section{Availability of data and materials}

The datasets generated and/or analyzed during the current study are available via the CBioportal online portal (http://www.cbioportal.org) and in the Genome-Phenome Archives EGAS00000000098 and phs000178 (https:// ega-archive.org/studies/EGAS00000000098 and https://ega-archive.org/studies/phs000178).

Ethics approval and consent to participate

Ethics approvals and consent for each cohort (METABRIC, TCGA, and MSKIMPACT) was obtained in the original studies.

\section{Consent for publication}

Not applicable

\section{Competing interests}

Since the first submission of the article, the corresponding author (SJJ) has moved employment to AstraZeneca, with no competing interests to declare. All other authors declare that they have no competing interests.

\section{Author details}

${ }^{1}$ Nottingham Breast Cancer Research Centre, Nottingham Biodiscovery Institute, School of Medicine, University of Nottingham, Nottingham, UK. ${ }^{2}$ Department of General Surgical Science, Gunma University Graduate School of Medicine, Gunma, Japan. ${ }^{3}$ Department of Breast Surgery, International University of Health and Welfare, Narita, Japan. ${ }^{4}$ School of Veterinary Medicine and Science, University of Nottingham, Nottingham, UK. ${ }^{5}$ Gene Regulation and RNA Biology Laboratory, School of Pharmacy, University of Nottingham, Nottingham, UK. ${ }^{6}$ Division of Oncogenomics, Oncode Institute, Netherlands Cancer Institute, Amsterdam, Netherlands. ${ }^{7}$ Womens Cancer Research Center, UPMC Hillman Cancer Center and Magee-Women Research Institute, Pittsburgh, PA, USA. ${ }^{8}$ Translational Medicine, Oncology Research and Development, AstraZeneca, Darwin Building, Milton, Cambridge, UK.

Received: 21 January 2020 Accepted: 26 July 2020

Published online: 11 August 2020

\section{References}

1. Martinez V, Azzopardi JG. Invasive lobular carcinoma of the breast: incidence and variants. Histopathology. 1979;3(6):467-88.

2. $\mathrm{Li} \mathrm{Cl}$, Anderson BO, Daling JR, Moe RE. Trends in incidence rates of invasive lobular and ductal breast carcinoma. JAMA. 2003;289(11):1421-4.

3. Li Cl, Daling JR. Changes in breast cancer incidence rates in the United States by histologic subtype and race/ethnicity, 1995 to 2004. Cancer Epidemiol Biomark Prev. 2007;16(12):2773-80.

4. Ferlay J, Colombet M, Soerjomataram I, Mathers C, Parkin DM, Pineros M, et al. Estimating the global cancer incidence and mortality in 2018: GLOBOCAN sources and methods. Int J Cancer. 2019:144(8):1941-53.

5. Pestalozzi BC, Zahrieh D, Mallon E, Gusterson BA, Price KN, Gelber RD, et al. Distinct clinical and prognostic features of infiltrating lobular carcinoma of the breast: combined results of 15 International Breast Cancer Study Group clinical trials. J Clin Oncol. 2008;26(18):3006-14.

6. Rakha EA, El-Sayed ME, Powe DG, Green AR, Habashy H, Grainge MJ, et al. Invasive lobular carcinoma of the breast: response to hormonal therapy and outcomes. Eur J Cancer. 2008;44(1):73-83.

7. Metzger Filho O, Giobbie-Hurder A, Mallon E, Gusterson B, Viale G, Winer EP et al. Relative effectiveness of letrozole compared with tamoxifen for patients with lobular carcinoma in the BIG 1-98 trial. J Clin Oncol. 2015; 33(25):2772-9.

8. Sikora MJ, Cooper KL, Bahreini A, Luthra S, Wang G, Chandran UR, et al. Invasive lobular carcinoma cell lines are characterized by unique estrogenmediated gene expression patterns and altered tamoxifen response. Cancer Res. 2014;74(5):1463-74.

9. NICE. Early and locally advanced breast cancer: diagnosis and management. Online: National Institute for Health and Care Excellence; 2018 [NICE Pathway]. Available from: https://www.nice.org.uk/guidance/ng101.

10. Cardoso F, Kyriakides S, Ohno S, Penault-Llorca F, Poortmans P, Rubio IT, et al. Early breast cancer: ESMO clinical practice guidelines for diagnosis, treatment and follow-updagger. Ann Oncol. 2019:30(8):1194-220.

11. Wolff AC, Hammond MEH, Allison KH, Harvey BE, Mangu PB, Bartlett JMS, et al. Human epidermal growth factor receptor 2 testing in breast cancer: American Society of Clinical Oncology/College of American Pathologists Clinical Practice Guideline Focused Update. J Clin Oncol. 2018;36(20):210522.

12. Denduluri N, Chavez-MacGregor M, Telli ML, Eisen A, Graff SL, Hassett MJ, et al. Selection of optimal adjuvant chemotherapy and targeted therapy for early breast Cancer: ASCO clinical practice guideline focused update. J Clin Oncol. 2018:36(23):2433-43.

13. Bose R, Kavuri SM, Searleman AC, Shen W, Shen D, Koboldt DC, et al. Activating HER2 mutations in HER2 gene amplification negative breast cancer. Cancer Discov. 2013;3(2):224-37.

14. Hanker A, Koch J, Ye D, Sliwoski G, Sheehan J, Kinch L, et al. Abstract PD305: Co-occurring gain-of-function mutations in HER2 and HER3 cooperate to enhance HER2/HER3 binding, HER-dependent signaling, and breast cancer growth. Cancer Res. 2019;79(4 Supplement):PD3-05-PD3. 
15. Eroglu Z, Tagawa T, Somlo G. Human epidermal growth factor receptor family-targeted therapies in the treatment of HER2-overexpressing breast cancer. Oncologist. 2014;19(2):135-50.

16. Hynes NE, MacDonald G. ErbB receptors and signaling pathways in cancer Curr Opin Cell Biol. 2009;21(2):177-84.

17. Junttila TT, Akita RW, Parsons K, Fields C, Lewis Phillips GD, Friedman LS, et al. Ligand-independent HER2/HER3/PI3K complex is disrupted by trastuzumab and is effectively inhibited by the PI3K inhibitor GDC-0941. Cancer Cell. 2009;15(5):429-40.

18. Mishra R, Patel H, Alanazi S, Yuan L, Garrett JT. HER3 signaling and targeted therapy in cancer. Oncol Rev. 2018;12(1):355.

19. Jaiswal BS, Kljavin NM, Stawiski EW, Chan E, Parikh C, Durinck S, et al. Oncogenic ERBB3 mutations in human cancers. Cancer Cell. 2013;23(5):60317.

20. Ping Z, Siegal GP, Harada S, Eltoum IE, Youssef $M$, Shen $T$, et al. ERBB2 mutation is associated with a worse prognosis in patients with $\mathrm{CDH} 1$ altered invasive lobular cancer of the breast. Oncotarget. 2016;7(49):8065563.

21. Ross JS, Wang K, Sheehan CE, Boguniewicz AB, Otto G, Downing SR, et al. Relapsed classic E-cadherin (CDH1)-mutated invasive lobular breast cancer shows a high frequency of HER2 (ERBB2) gene mutations. Clin Cancer Res. 2013;19(10):2668-76.

22. Griffith OL, Spies NC, Anurag M, Griffith M, Luo J, Tu D, et al. The prognostic effects of somatic mutations in ER-positive breast cancer. Nat Commun. 2018;9(1):3476

23. Ross JS, Gay LM, Wang K, Ali SM, Chumsri S, Elvin JA, et al. Nonamplification ERBB2 genomic alterations in 5605 cases of recurrent and metastatic breast cancer: an emerging opportunity for anti-HER2 targeted therapies. Cancer. 2016;122(17):2654-62.

24. Desmedt C, Zoppoli G, Gundem G, Pruneri G, Larsimont D, Fornili M, et al. Genomic characterization of primary invasive lobular breast cancer. J Clin Oncol. 2016;34(16):1872-81.

25. Ciriello G, Gatza ML, Beck AH, Wilkerson MD, Rhie SK, Pastore A, et al. Comprehensive molecular portraits of invasive lobular breast cancer. Cell. 2015;163(2):506-19.

26. Pereira B, Chin SF, Rueda OM, Vollan HK, Provenzano E, Bardwell HA, et al. The somatic mutation profiles of 2,433 breast cancers refines their genomic and transcriptomic landscapes. Nat Commun. 2016;7:11479.

27. Razavi P, Chang MT, Xu G, Bandlamudi C, Ross DS, Vasan N, et al. The genomic landscape of endocrine-resistant advanced breast cancers. Cancer Cell. 2018;34(3):427-38 e6.

28. Kadota K, Nakai Y, Shimizu K. A weighted average difference method for detecting differentially expressed genes from microarray data. Algorithms Mol Biol. 2008;3:8.

29. Curtis C, Shah SP, Chin SF, Turashvili G, Rueda OM, Dunning MJ, et al. The genomic and transcriptomic architecture of 2,000 breast tumours reveals novel subgroups. Nature. 2012;486(7403):346-52.

30. Ferrari A, Vincent-Salomon A, Pivot X, Sertier AS, Thomas E, Tonon L, et al. A whole-genome sequence and transcriptome perspective on HER2-positive breast cancers. Nat Commun. 2016;7:12222.

31. Desmedt C, Haibe-Kains B, Wirapati P, Buyse M, Larsimont D, Bontempi G, et al. Biological processes associated with breast cancer clinical outcome depend on the molecular subtypes. Clin Cancer Res. 2008;14(16):5158-65.

32. Sareyeldin RM, Gupta I, Al-Hashimi I, Al-Thawadi HA, Al Farsi HF, Vranic S, et al. Gene expression and miRNAs profiling: function and regulation in human epidermal growth factor receptor 2 (HER2)-positive breast cancer. Cancers (Basel). 2019;11(5):646.

33. Creighton CJ, Hilger AM, Murthy S, Rae JM, Chinnaiyan AM, El-Ashry D. Activation of mitogen-activated protein kinase in estrogen receptor alphapositive breast cancer cells in vitro induces an in vivo molecular phenotype of estrogen receptor alpha-negative human breast tumors. Cancer Res. 2006;66(7):3903-11.

34. Rees MG, Seashore-Ludlow B, Cheah JH, Adams DJ, Price EV, Gill S, et al. Correlating chemical sensitivity and basal gene expression reveals mechanism of action. Nat Chem Biol. 2016;12(2):109-16.

35. Rajapakse VN, Luna A, Yamade M, Loman L, Varma S, Sunshine M, et al. CellMinerCDB for integrative cross-database genomics and pharmacogenomics analyses of cancer cell lines. iscience. 2018;10:247-64.

36. Basu A, Bodycombe NE, Cheah JH, Price EV, Liu K, Schaefer Gl, et al. An interactive resource to identify cancer genetic and lineage dependencies targeted by small molecules. Cell. 2013;154(5):1151-61.
37. Ma CX, Bose R, Gao F, Freedman RA, Pegram MD, Blackwell K, et al. Phase II trial of neratinib for HER2 mutated, non-amplified metastatic breast cancer (HER2(mut) MBC). J Clin Oncol. 2016;34(15 suppl):516.

38. Syed BM, Green AR, Paish EC, Soria D, Garibaldi J, Morgan L, et al. Biology of primary breast cancer in older women treated by surgery: with correlation with long-term clinical outcome and comparison with their younger counterparts. Br J Cancer. 2013;108(5):1042-51.

\section{Publisher's Note}

Springer Nature remains neutral with regard to jurisdictional claims in published maps and institutional affiliations.
Ready to submit your research? Choose BMC and benefit from:

- fast, convenient online submission

- thorough peer review by experienced researchers in your field

- rapid publication on acceptance

- support for research data, including large and complex data types

- gold Open Access which fosters wider collaboration and increased citations

- maximum visibility for your research: over $100 \mathrm{M}$ website views per year

At BMC, research is always in progress.

Learn more biomedcentral.com/submissions 